\title{
From Propped up to Prone: A Paradigm Shift in the Management of Severe COVID Pneumonia!
}

\author{
Arindam Choudhury $^{10}$ Deva Senathipati ${ }^{2}$ \\ ${ }^{1}$ Department of Cardiac Anesthesia and Critical Care, Cardiothoracic \\ and Neurosciences Centre, All India Institute of Medical Sciences, \\ New Delhi, India \\ 2 Department of Radiology, All India Institute of Medical Sciences, \\ New Delhi, India \\ J Card Crit Care 2021;5:265-267.
}

Address for correspondence Arindam Choudhury, MD, Department of Cardiac Anesthesia and Critical Care, Cardiothoracic and Neurosciences Centre, All India Institute of Medical Sciences, New Delhi, India - 110029 (e-mail: archymd@gmail.com).

\begin{abstract}
Keywords

- COVID pneumonia

- COVID19

- hypoxia

- prone ventilation

- proning

Coronavirus disease (COVID) pneumonia in the recent times has shown us the importance of prone positioning during the management of moderate to severe hypoxia. In fact, we have rediscovered the benefits of this positional maneuver in coping with the "oxygen crisis" during the second wave of this dreaded pandemic. While we (the clinicians) derive benefits of this postural therapy for treating our patients, it is imperative for us to know the underlying mechanisms behind improvement in oxygenation in mild to moderate cases of COVID pneumonia. In this brief communication, we try to decipher the miracles of this great "life-saver."
\end{abstract}

It is indeed a challenge for all the critical care specialists to race against time while managing severe coronavirus disease (COVID) pneumonia caused by severe acute respiratory syndrome coronavirus 2 virus (in $\sim 5 \%$ ). On one hand we are constantly under pressure trying to maintain patients' oxygen saturation above the cutoff range, while we continue to monitor the patient maintaining him in prone position on the other hand. From time immemorial, we were taught that the position of choice in a critical care setup is the 30-degree propped up position. Traditionally, we were taught how head-up position in a critically ill patient not only benefits the respiratory mechanics, but it also tends to protect the lung from microaspirations and consequent ventilator-associated pneumonia. All these concepts seem irrelevant for the management of COVID pneumonia associated with severe acute respiratory distress syndrome (ARDS). Equally intriguing is the fact that many of these patients' oxygen saturation deteriorates when they try to sit up for having their meals.
Therefore, it is quite but natural for an inquisitive mind to ask what has changed: the dictum or the disease. In this case, it is the latter which has changed. Let me try to explain.

Patients of COVID pneumonia needing intensive care unit (ICU) care suffers from a severe form of acute lung injury and more often severe ARDS. Their $\mathrm{PaO}_{2}: \mathrm{FiO}_{2}(\mathrm{P} / \mathrm{F})$ ratio is usually less than 150 and at times $<100$. When we analyzed the computed tomography (CT) chest of these patients, we found a characteristic ground-glass pattern predominantly involving the periphery and the posterior aspect of the lower lobes of the lungs. ${ }^{1}$

Also, when we delve into the pathogenesis of COVID-19 pneumonia, we find that there is progressive fibrosis of air spaces following alveolitis, pulmonary edema, and atelectasis. All these changes lead to increased right-to-left shunting. Also, there are increased thrombotic events due to "microthrombosis" in the capillary beds surrounding each alveolus as well as endothelial inflammation and pleural effusion. All
DOI https://doi.org/ $10.1055 / \mathrm{s}-0041-1741494$. ISSN 2457-0206.
(C) 2022. Official Publication of The Simulation Society (TSS), accredited by International Society of Cardiovascular Ultrasound (ISCU). All rights reserved.

This is an open access article published by Thieme under the terms of the Creative Commons Attribution-NonDerivative-NonCommercial-License, permitting copying and reproduction so long as the original work is given appropriate credit. Contents may not be used for commercial purposes, or adapted, remixed, transformed or built upon. (https://creativecommons.org/ licenses/by-nc-nd/4.0/)

Thieme Medical and Scientific Publishers Pvt. Ltd., A-12, 2nd Floor, Sector 2, Noida-201301 UP, India 

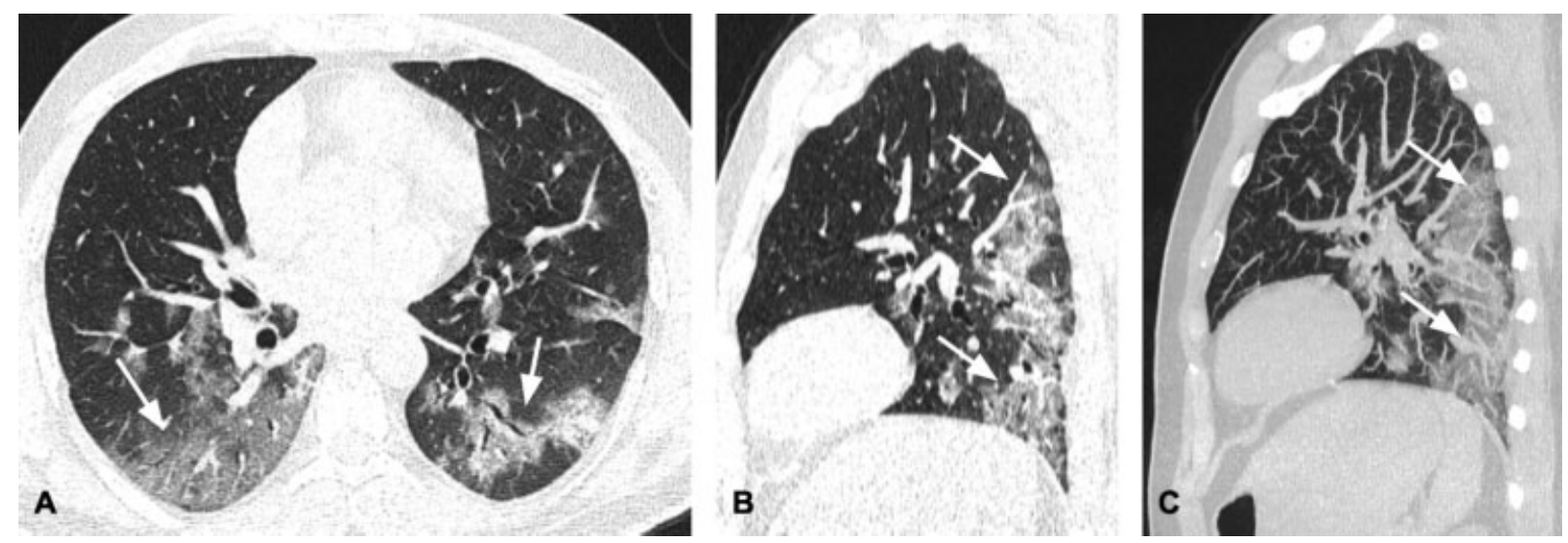

Fig. 1 (A-C) Axial (A) and sagittal (B) computed tomography (CT) scan images in lung window and sagittal thin maximum intensity projection (MIP) image in a patient diagnosed with COVID-19 pneumonia showing ground-glass opacities and consolidation (arrows) predominantly involving the posterior segments of bilateral lower lobes. The upper lobes and the nondependent areas of bilateral lungs are relatively free of disease.

these changes lead to increased dead space ventilation and increased diffusion barrier. ${ }^{2}$ Thus, patients with severe COVID pneumonia experience a lot of ventilation-perfusion (V/Q) mismatch leading to extreme hypoxemia (often causing type 2 respiratory failure). This massive $\mathrm{V} / \mathrm{Q}$ mismatch occurs due to a combination of dead space ventilation and pathologic shunting of deoxygenated blood in the affected areas of the lung.

As we have seen from CT scans, the majority of patients who were admitted in the ICU have bilateral lower lobe and posterior surface involvement (-Fig. 1). When patients lie supine or try to sit upright, there is a gravity-dependent shift of pulmonary blood flow toward posterior zones or lower lobes capillaries, respectively. ${ }^{3}$ However, due to microangiopathy and microthrombi in these vessels causing congestions of capillaries coupled with exaggerated alveolar edema (increasing the hydrostatic pressure and/or pleural pressure decreasing the alveolar compliance and distancing the gaseous phase from the already compromised capillary blood flow) jeopardize gas exchanges. As $\mathrm{CO}_{2}$ is 20 times more diffusible than $\mathrm{O}_{2}$, most patients with no prior lung disease escaped hypercarbia. ${ }^{4}$ But, in patients with preexisting obstructive lung diseases, obese, and elderly individuals, some $\mathrm{CO}_{2}$ retention was seen along with severe hypoxemia. However, a subset of very sick patients with COVID pneumonia whose $\mathrm{PaCO}_{2}$ did not decrease with prone position are considered "nonresponders" and they had the worst outcome.

In an otherwise healthy lung in upright position, $\mathrm{V} / \mathrm{Q}$ occurs in accordance with the principles outlined by West et al. ${ }^{5}$ Depending on the vertical height above or below the heart, the alveoli tend to overdistend or collapse deciding between dead space ventilation or minimal physiological shunting (-Fig. 2). This is purely governed by the alveolar capillary pressures within the physiological range so that the $\mathrm{V} / \mathrm{Q}$ is perfectly matched and the individual maintains normoxia. However, a slight increase in dead space ventilation in a diseased lung in COVID pneumonia (vide supra) leads to a severe degree of $\mathrm{V} / \mathrm{Q}$ mismatch which is already compro- mised due to other pathophysiologic mechanisms outlined above. On the contrary, these patients generally do better in terms of gas exchange when they are in prone position because the entire anterior portion of both lungs is usually spared of the disease. In prone position, relatively healthier lungs are available for gas exchange and the $\mathrm{V} / \mathrm{Q}$ mismatch is minimal as the dependent lung vertical height is not much lowered from the heart (as heart is anteriorly placed in the mediastinum). Moreover, prone positioning (proning) tend to relieve cardiac compression on the dorsal lung $(\mathrm{L}>\mathrm{R})$ regions as it lies directly over the sternum and this compression is redirected to only a miniscule portion of the ventral lung (dependent) regions improving the lymphatic drainage, lung compliance, and efficiency of gas exchange. ${ }^{6}$ The alveolar capillary pressures are not raised enough to create shunting unless there is high intrathoracic pressure due to very low compliance (H-phenotype). ${ }^{2}$ In our experience, most patients with severe ARDS associated with COVID pneumonia (L-phenotype) have responded favorably to "proning" with improvements in their $\mathrm{PaO}_{2}$ (and PF ratio) barring a few patients with preexisting postpartum hemorrhage due to valvular heart diseases.

Finally, COVID-19 has led us to reinvent and redefine ICU care of critically ill patients which is predominantly a respiratory disorder. Many patients are comfortable lying prone for long hours sipping fluids/liquid diets while watching their oxygen saturations improve. This is a tremendous morale booster and is instrumental for a large number of our patients whom we have been able to transfer to a low dependency unit or even discharge home. We have offered prone position even in patients with persistently low PF ratio and they were resuscitated in prone position during cardiac arrest as they were on controlled mandatory ventilation with endotracheal tube in situ. Thus, prone position has become the standard of care in COVID ICUs with newer adjustments to our work flow.

\section{Conflict of Interest}

None declared. 

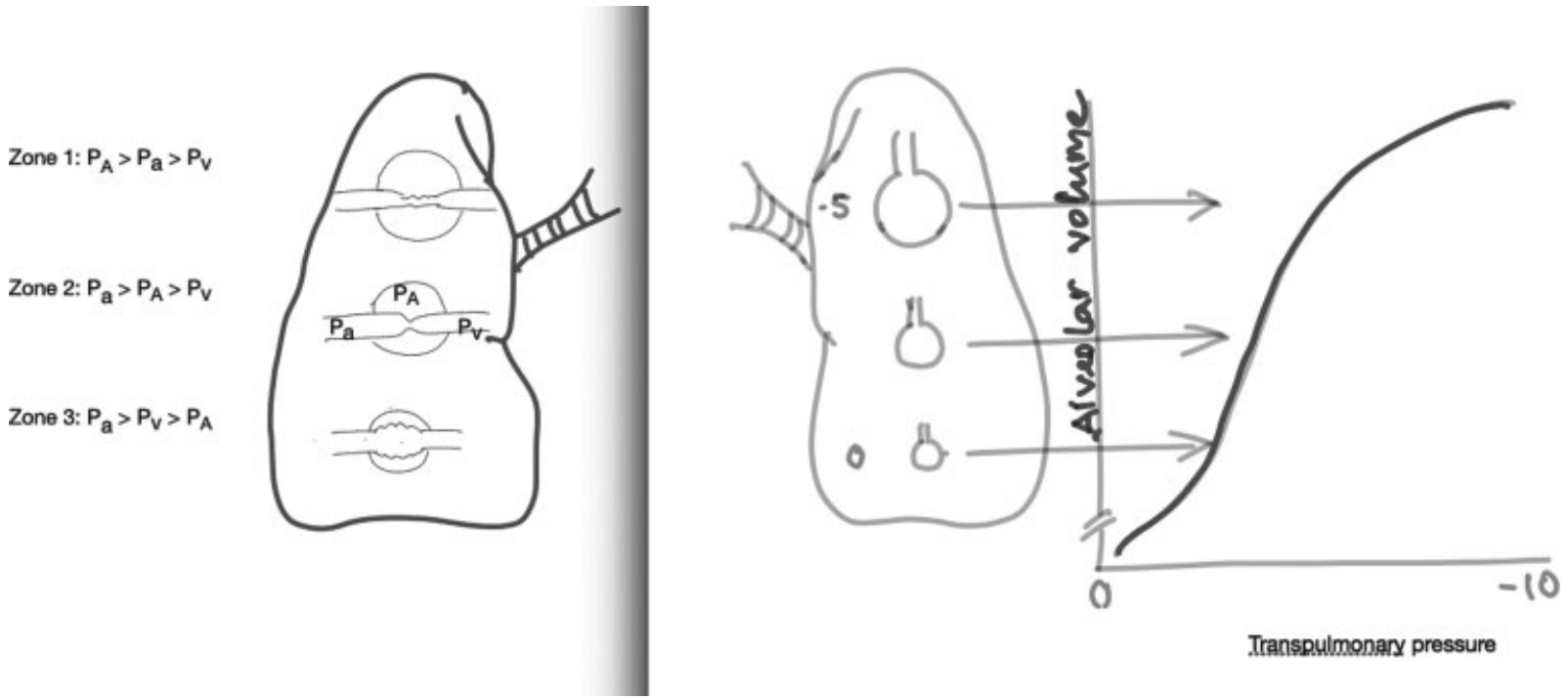

Fig. 2 Distribution of perfusion is depicted in upright position by the three-zone model (A); right panel showing the effect of gravity on transpulmonary pressure in a healthy individual affecting the alveolar compliance (B). This effect is exaggerated in acute respiratory distress syndrome (ARDS) due to increasing mass of inflamed and edematous lung (due to the hydrostatic pressure imposed by the weight of the affected tissue) toward the dependent region. $\mathrm{P}_{\mathrm{A}}$, alveolar pressure; $\mathrm{P}_{\mathrm{a}}$, arterial pressure; $\mathrm{P}_{\mathrm{v}}$, venous pressure.

\section{References}

1 Pan F, Ye T, Sun P, et al. Time course of lung changes on chest CT during recovery from 2019 novel coronavirus (COVID-19) pneumonia. Radiology 2020;295(03):715-721

2 Osuchowski MF, Winkler MS, Skirecki T, et al. The Covid-19 puzzle: deciphering pathophysiology and phenotype of a new disease entity. Lancet Respir Med 2021;9(06):622-664

3 Hughes JMB. Distribution of pulmonary blood flow. In: Crystal RG, West JB, Weibel ER, Barnes PJ, eds. The Lung: Scientific Foundations. 2nd ed. Philadelphia: Lippincott-Raven; 1997:1523-1536
4 Ottestad W, Søvik S. COVID-19 patients with respiratory failure: what can we learn from aviation medicine? Br J Anaesth 2020;125 (03):e280-e281

5 West JB, Dollery CT, Naimark A. Distribution of blood flow in isolated lung: relation to vascular and alveolar pressures. J Appl Physiol 1964;19:713-724

6 Albert RK, Hubmayr RD. The prone position eliminates compression of the lungs by the heart. Am J Respir Crit Care Med 2000;161 (05):1660-1665 